\title{
Profile characterization and temperature dependence of droplet control on textured surfaces
}

\author{
FENG RuoTao $^{1,2}$, WU XueDong $^{1 *} \&$ XUE QunJi ${ }^{1}$ \\ ${ }^{1}$ Ningbo Institute of Material Technology and Engineering, Chinese Academy of Sciences, Ningbo 315201, China; \\ ${ }^{2}$ Graduate University of the Chinese Academy of Sciences, Beijing 100049, China
}

Received July 14, 2010; accepted November 22, 2010; published online May 12, 2011

\begin{abstract}
Droplet control on textured surfaces has attracted much attention in the fields of drag reduction and high-efficiency microreactors. We investigated the profile characterization and temperature dependence of droplet control between wetting and non-wetting on a series of textured silicon surfaces. Surfaces were fabricated using photolithography, and the temperature-dependent wettability of the single phase regime droplets was characterized using dynamic contact angle measurements. The experiments demonstrated that the profile of textured surfaces characterizes the droplets Leidenfrost point. The Leidenfrost point is proportional to the micro-pillar size and rectangular wave-length of the textured surface and is inversely proportional to ambient temperature. The Leidenfrost droplets moved freely on the textured surface, almost without hysteresis. Droplets could be directed on the surface of microreactors with a low driving force.
\end{abstract}

textured surface, Leidenfrost droplet, droplet control, drag reduction, microreactors

Citation: $\quad$ Feng R T, Wu X D, Xue Q J. Profile characterization and temperature dependence of droplet control on textured surfaces. Chinese Sci Bull, 2011, 56: 1930-1934, doi: 10.1007/s11434-010-4294-x

Microelectronic mechanical systems have recently been developing rapidly across many fields. Microfluidics is essentially a branch of microelectronic mechanical systems and has applications in biochemical analysis, blood tests, and microelectronic cooling systems [1]. It is becoming clear that the traditional methods for doing chemistry are not sustainable and must be changed. Droplet-based microreactor technologies offer a solution because they are inherently less wasteful than traditional methods and because they provide unprecedented reaction control. The small size of microreactors provides complete mixing and precise temperature control, thus avoiding hot spots. It also offers better regioselectivity, chemical selectivity, and yield for many chemical reactions with minimal byproducts [2-7]. With the development of better droplet control models, microreactors have attracted much attention from the fundamental and applied scientists in biology and chemistry $[8,9]$.

*Corresponding author (email: xdwu@nimte.ac.cn)
Microreactors still require improvements on many issues, including dynamic flow development, precise droplet control, rectifying incompatibilities with reagents and intermediates, and the removal of side products. Hence, precise droplet control is a core issue. Methods for manipulating droplets are continuously being developed to take advantage of physical principles. Examples include hydrodynamics, acoustic waves [10] or mechanical vibrations [11], electrokinetics [12,13], thermal gradient effects [14], Marangoni effects [15-17], and surface chemistry [18,19]. Such developments will lead to further applications, especially for thermal gradient systems where a droplet deposited on a Leidenfrost ratchet-like surface has achieved speeds of 5 $\mathrm{cm} / \mathrm{s}$ over distances of up to $1 \mathrm{~m}$, without any external power. For a Leidenfrost droplet, a smooth hysteresis arises from a lubricating vapor layer separating the droplet from the supporting surface [20]. In 1756, Leidenfrost discovered that a droplet could maintain its motion and be sustained for a long time on heated surfaces by a vapor layer, and this 
became known as self-lubrication of the Leidenfrost droplet. However, it is unknown how the profile of rough surfaces affects the Leidenfrost droplet. Advances in research of Leidenfrost droplets will help lead to precise control of thermal and motion states in droplet-based microreactors.

Here, we report the temperature dependence of droplet control on a textured surface. The textured surfaces have been constructed from parallel square-pillars fabricated by the photolithography of silicon plates. The temperaturedependent wettability of single-phase regime droplets was characterized on these surfaces using dynamic contact angle measurements. With increasing temperature, the microliter-sized droplets showed single-phase, nucleate-boiling, transition-boiling, and film-boiling regimes. Droplets have been called Leidenfrost droplets within the film-boiling regime. The Leidenfrost droplets moved freely on an inclined textured surface (inclination angle of $2^{\circ}$ ), and droplets could be directed with a low driving force. Our experiments have characterized the effect of textured surface profile on the droplet's Leidenfrost point (LFP). The LFP is proportional to micro-pillar size and rectangular wavelength of the textured surfaces and inversely proportional to ambient temperature.

\section{Experimental}

\subsection{Design of the textured surface in microreactors}

Microreactors have been constructed from various materials, such as silicon, quartz, glass, metals, and polymers. Various fabrication methods are available, including photolithography, hot embossing, powder blasting, injection molding and laser micro forming, the choice of which depends on the material being used [21]. Microreactors have often been designed with many micro channels for use in a two-phase fluid system. To our knowledge, there have been few details published concerning microreactors prepared from textured plates. If such a microreactor could induce the formation of self-lubricating Leidenfrost droplets, it would be useful for controlling the motion and heat management of the droplets.

\subsection{Fabrication of the textured surface}

Textured surfaces with square pillars of different dimensions were made from silicon wafers. The silicon was etched using lithography (Karl Suss MA6, Shipley LC100A $1 \mu \mathrm{m})$ and inductively coupled plasma techniques to form square micropillar arrays. This is depicted scheme schematically in Figure 1. The regular textured surfaces consisted of a micro square pillar array, characterized by pillar size $d \times d$, height $H$, and pillar spacing $w$. The rough surface was designed in a regular arrangement with a variable roughness gradient, by controlling $d$ and $w$ (parameters of (a)

(b)
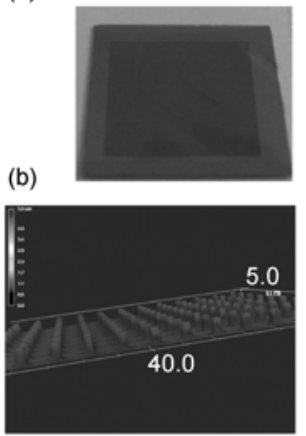

(c)

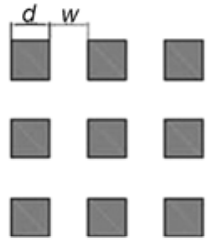

(d)

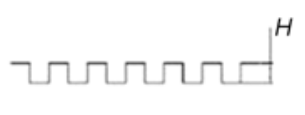

Figure 1 (a) Photograph of the textured surface sample; (b) A 3D image of the textured surface (3D laser scanning microscope dimensions system, Keyence VK-9700). Plan and cross-section schematic views of the square pillar geometry are shown in (c) and (d), respectively.

the textured surface are shown in Table 1). The height was kept constant for all the micropillars at $5 \mu \mathrm{m}$.

\subsection{Characterization of droplets on the textured surface}

The dynamic contact angle and morphology of droplets on the textured surface were recorded using a camera of Dataphysics contact angle measuring system (OCA-20, Germany, 200 frames/s). A dataphysics TC/TEC 700 electrical thermal chamber was used to control the surface temperature. Droplets were deposited by a Hamilton DS 500/GT (USA) precision syringe. Silicon plates were ultrasonically cleaned in ethanol before use. Deionized water was used in all experiments and the water was frequently replaced to ensure consistent fluidic conditions.

Table 1 Parameters profiling the textured surface

\begin{tabular}{lllll}
\hline \multicolumn{2}{c}{ Group } & $d(\mu \mathrm{m})^{\mathrm{a})}$ & $w(\mu \mathrm{m})^{\mathrm{a})}$ & $f^{\mathrm{b})}$ \\
\hline \multirow{4}{*}{ A } & A1 & 2.5 & 2.5 & 0.25 \\
& A2 & 2.5 & 3.09 & 0.2 \\
& A3 & 2.5 & 4 & 0.15 \\
& A4 & 2.5 & 5.4 & 0.1 \\
& A5 & 2.5 & 8.7 & 0.05 \\
& B1 & 5 & 5 & 0.25 \\
& B2 & 5 & 6.2 & 0.2 \\
& B3 & 5 & 7.9 & 0.15 \\
& B4 & 5 & 10.8 & 0.1 \\
& B5 & 5 & 17.4 & 0.05 \\
& C1 & 10 & 10 & 0.25 \\
& C2 & 10 & 12.4 & 0.2 \\
C & C3 & 10 & 15.84 & 0.15 \\
& C4 & 10 & 21.6 & 0.1 \\
& C5 & 10 & 34.7 & 0.05 \\
& D1 & 50 & 50 & 0.25 \\
\hline
\end{tabular}

a) Arithmetic mean, b) $f=d^{2} /\left(d^{2}+w^{2}\right)$. 


\section{Results and discussion}

With increasing temperature of the textured surface, a transformation from wetting to dewetting of the droplet properties was observed. This progressed through four distinct heat transfer regimes, namely individual single phase, nucleate boiling, transition boiling, and film boiling regimes (Figure 2). Only the film-boiling regime provided the droplet with the ability for motion, lubricating the motion using the evaporation film between the droplet and surface. This droplet took longer to evaporate than the other droplets at high temperature. The surface temperature during the droplet state transformation from transition boiling to film boiling is termed the LFP. Once in the film boiling regime, the droplet is known as the Leidenfrost droplet. Leidenfrost droplets offer the potential for accurate droplet control in microreactors, given the self-lubricating and long-surviving properties of such droplets at high temperature. Leidenfrost droplets may also offer an ideal micro-environment to sustain a constant solute concentration during reaction, with the inert carrier gas removing surplus evaporation from the solution.

Gas-liquid-solid interfaces of droplets on a textured surface can be continuous in the single-phase regime. The temperature-dependent wettability of single-phase regime droplets was characterized using dynamic contact angle measurements. In this regime, heat from the surface was conducted through the liquid film and dissipated by evaporation at the liquid-gas interface. In the nucleate boiling regime, vapor bubbles were produced, and the corresponding heat flux increased dramatically. Droplet lifetime decreased and tended to a minimum at the critical heat flux point. We traced the droplet state in these two regimes using two different profiled textured silicon surfaces (A1 and D1, Table 1) and dynamic contact angle measurements at three different temperatures $\left(72^{\circ} \mathrm{C}, 92^{\circ} \mathrm{C}, 122^{\circ} \mathrm{C}\right)$, as shown in Figure 3. With increasing temperature, droplet lifetime

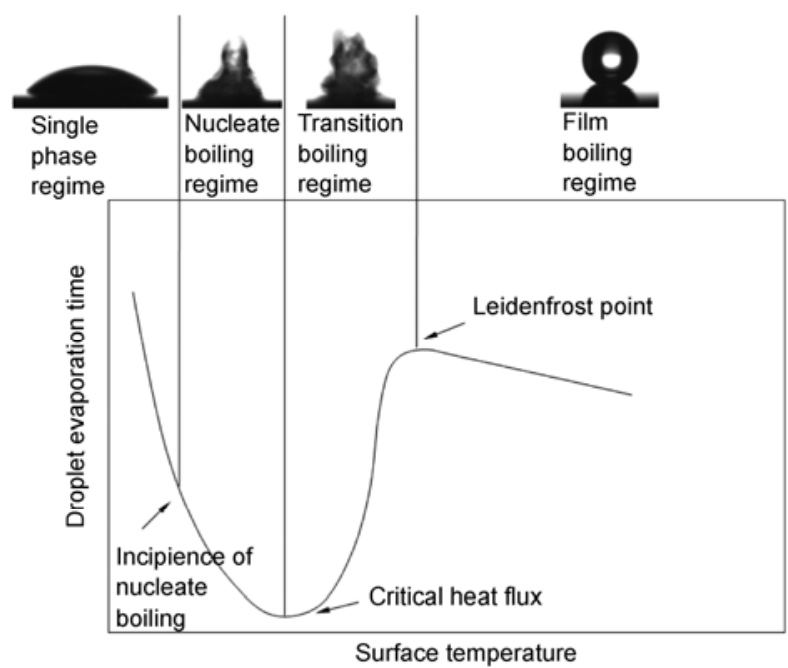

Figure 2 Curve showing droplet evaporation on a hot textured silicon surface.
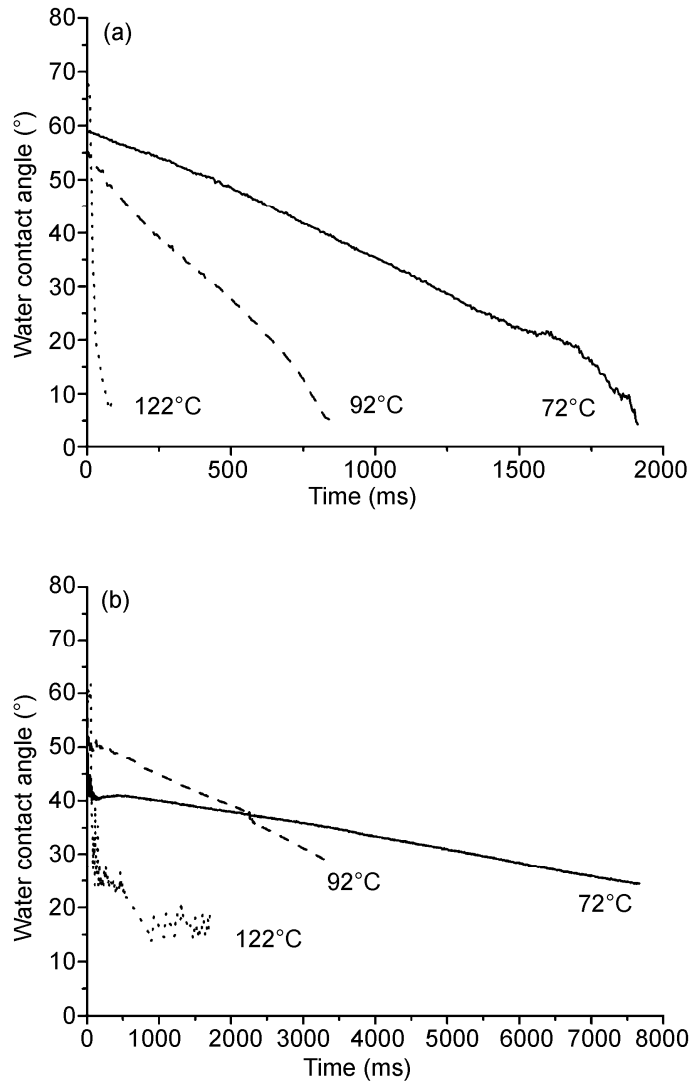

Figure 3 Dynamic contact angle curves for droplets on textured surfaces. (a) A1 and (b) D1 textured surfaces (see Table 1).

quickly decreased. Droplets evaporated faster on the smaller pillar surface. The droplet profile decreased in the single phase regime, and the contact angle gradually declined. Droplets could not maintain a continuous profile at $122^{\circ} \mathrm{C}$, because vapor production increased in the nucleate boiling regime. These experiments demonstrated that the heat conduction of the textured silicon surfaces could be increased by increasing the surface roughness. It effectively shortened the wave-length of the surface micro pattern.

With increasing surface temperature, the droplet progressed into the transition regime. Compared with the first two regimes, the surface showed a distinctive heat flux route between the solid-liquid interface. In the transition regime, a non-continuous insulating vapor layer developed beneath portions of the droplet, leading to a reduced evaporation rate and increased drop lifetime. Heat conduction decreased and heat emission increased. At the upper end of the transition boiling regime (LFP), the vapor layer grew substantially to prevent any significant contact between the droplet and surface. Here, droplet evaporation time reached a maximum. Above the LFP, the droplets remained separated from the surface by a thin vapor layer, through which heat was transmitted.

Any rough surface could potentially show characteristics of heat emission. Bernarain et al. reported that the LFP 
temperature was sensitive to polished, particle blasted and rough sanded surface finishes and their respective average roughness values were 97,970 and $2960 \mathrm{~nm}$ [22]. Their study was limited to finishing methods and could not provide an accurate relationship between the surface microgeometry and droplet LFP. In contrast, our study investigated controlling the Leidenfrost droplet on a series of three textured silicon surfaces at a temperature of $23^{\circ} \mathrm{C}$. Figure 4(a) shows that the droplet LFP temperature depended on the pillar geometry of the textured surface. In the same pillar size group, the LFP temperature decreased with the area ratio ( $f$, the rate apparent surface to project surface) of the surface. For the same area ratio, the LFP temperature increased with increasing pillar size. We speculate that the LFP temperature change resulted from various hot spots distributed on the surface, shown schematically in Figure 5. The short wave length of the micro pattern led to greater heat emission. The temperature of the top of the pillar will also be higher than that of the bottom of the spaces between. The top of the textured surface then adopted a thermal distribution pattern, which is representative of the orthogonal rectangular wave of the textured silicon surface. The distribution of the hot micro-sites was directly related to the vapor flow. Conversely, the vapor torrent affected the stability of the evaporated film under the droplet. Lastly, the droplet was broken up, and the LFP temperature increased.
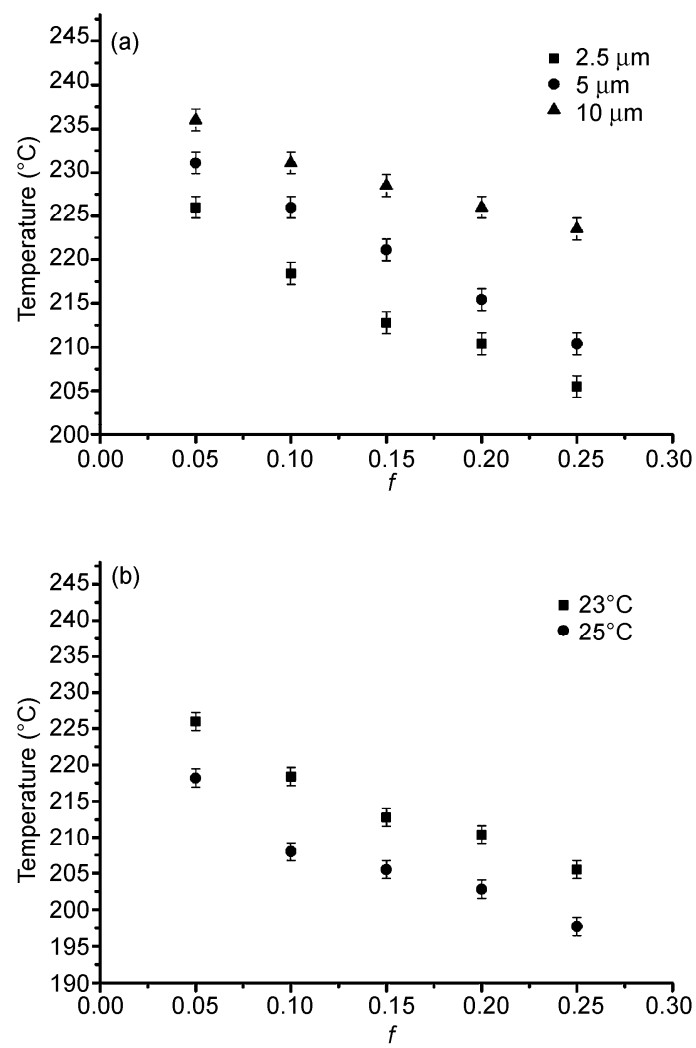

Figure 4 (a) Dependence of LFP temperature on droplet geometry, where $f$ is the droplet area ratio; (b) effect of ambient temperature on the droplet LFP temperature. The textured surface was from series A (Table 1).

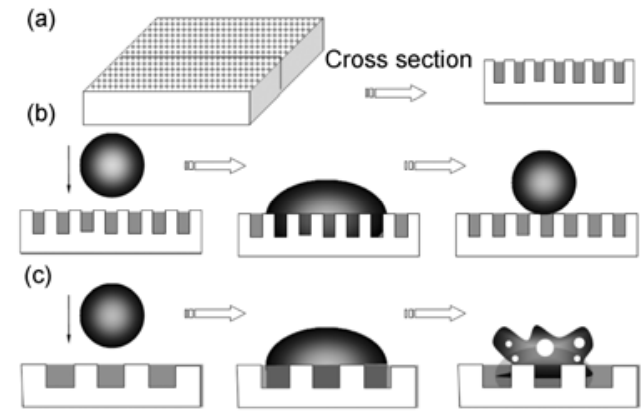

Figure 5 (a) The scheme of hot spots distributions on a textured surface; (b) effect of the short-wave surface on droplet configuration; (c) effect of the long-wave surface on droplet configuration.

Droplet LFP temperature depended on the surface geometry and also on the ambient temperature (Figure 4b). As ambient temperature increased from $23^{\circ} \mathrm{C}$ to $25^{\circ} \mathrm{C}$, the LFP temperature decreased on $2.5 \mu \mathrm{m}$ pillar series textured surface. This is because the droplets reduced thermal requirements for constructing the evaporated film in the high temperature environment.

Figure 6(a) and 6(b) show the stickiness of the droplet on a textured surface at $50^{\circ} \mathrm{C}$, which was lower than the LFP temperature. Figure 6(c) and (d) show the non-stickiness and self-lubricating motion of the Leidenfrost droplet. As the film boiling regime progressed, droplet mobility was assisted by the evaporated film. The Leidenfrost droplet could vibrate freely on the horizontal textured surface (Figure 6(c)). It could also move smoothly on the inclined textured surface (Figure 6(d)). A microreactor treated with different textured surfaces may satisfy different requirements for chemical reaction temperature, and so could be suitable for different solvent environments. Leidenfrost supercritical carbon dioxide droplets could also be used in the microreactor system. This research can contribute to building an ideal green system for the organic synthesis and drug discovery, as it would reduce expense, space, and labor requirements.

\section{Conclusions}

In this study, we have presented a thermal-assisted microreactor model with controllable Leidenfrost droplets on textured surfaces that could be used to save energy. The experiments demonstrated that the profile of the textured surfaces characterizes the LFP of droplets. The LFP of droplets was proportional to the micro-pillar size and rectangular wave-length of the textured surfaces, and inversely proportional to ambient temperature. The self-lubrication of the Leidenfrost droplet minimizes drag in microreactors. Based on our study, the profile characterization and temperature dependence of droplet control could be promising for microreactor development. The availability of microreactors 
(a)

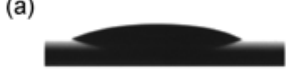

(b)

(c)
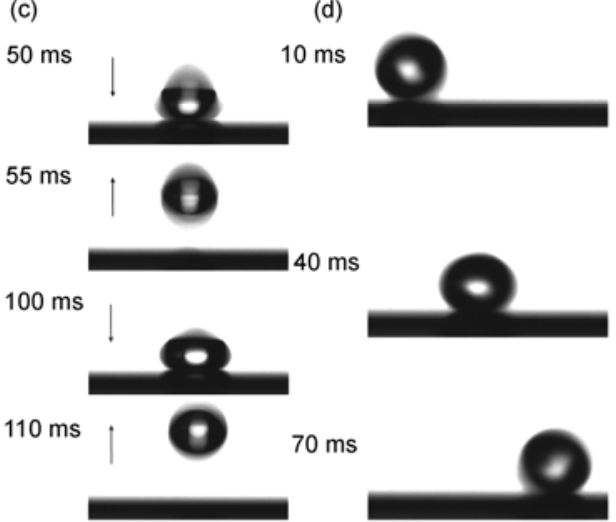

Figure 6 (a) Droplet shape on a horizontal A1 textured surface at $50^{\circ} \mathrm{C}$; (b) droplet shape on an inclined $\mathrm{A} 1$ textured surface at $50^{\circ} \mathrm{C}$, with a $30^{\circ}$ angle of inclination; (c) the vibration of the Leidenfrost droplet on a horizontal A1 textured surface at 50, 55, 100 and $110 \mathrm{~ms}$; (d) the motion of the Leidenfrost droplet on an inclined A1 textured surface at 10, 40 and $70 \mathrm{~ms}$, with a $2^{\circ}$ angle of inclination.

with different textured surfaces may broaden their application in the chemical reactions over wide temperature ranges. Accumulated knowledge on textured surfaces will advance the design of future microreactors and benefit the chemical industry, building a greener world.

This work was supported by the National Nature Science Foundation of China (50775212 and 20306014) and the Outstanding Talent Recruiting Program (2009A31004) dedicated to Academician Q. J. Xue from Ningbo Municipal Government.

1 Squires T M, Quake S R. Microfluidics: Fluid physics at the nanoliter scale. Rev Mod Phys, 2005, 77: 977-1026

2 Broadwater S J, Roth S L, Price K E, et al. One-pot multi-step synthesis: A challenge spawning innovation. Org Biomol Chem, 2005, 3: 2899
3 Gritzner G, Kreysa G. Nomenclature, symbols and definitions in electrochemical engineering. J Electroanal Chem, 1993, 360: 351-362

4 Jähnisch K, Hessel V, Löwe H, et al. Chemistry in microstructured reactors. Angew Chem Int Ed, 2004, 43: 406-446

5 Watts P, Haswell S J. The application of micro reactors for organic synthesis. Chem Soc Rev, 2005, 34: 235-246

6 Wörz O, Jäckel K P, Richter Th, et al. Microreactors, a new efficient tool for optimum reactor design. Chem Eng Sci, 2001, 56: 10291033

7 Fletcher P D I, Haswell S J, Pombo-Villar E, et al. Micro reactors: Principles and applications in organic synthesis. Tetrahedron, 2002, 58: 4735-4757

8 Song H, Chen D L, Ismagilov R F. Reactions in droplets in microfluidic channels. Angew Chem Int Ed, 2006, 45: 7336-7356

9 Mason B P, Price K E, Steinbacher J L, et al. Greener approaches to organic synthesis using microreactors technology. Chem Rev, 2007, 107: 2300-2318

10 Daniel S, Chaudhury M K, de Gennes P G. Vibration-actuated drop motion on surfaces for batch microfluidic process. Langmuir, 2005, 21: 4240-4248

11 Noblin X, Kofman R, Celestini F. Ratchetlike motion of a shaken drop. Phys Rev Lett, 2009, 102: 194504

12 Jorgenson J W, Lukacs K D. Zone electrophoreisis in open-tubular glass capillaries. Anal Chem, 1981, 53: 1298-1302

13 Li D. Electrokinetics in Microfluidics. Vol. 2. Amsterdam: Elsevier, 2004

14 Linke H, Alemán B J, Melling L D, et al. Self-propelled Leidenfrost droplets. Phys Rev Lett, 2006, 96: 154502

15 de Gennes P G, Brochard-Wyart F, Quéré D. Capillarity and Wetting Phenomena. New York: Springer, 2003

16 Kotz K T, Noble K A, Faris G W. Optical Microfluidics. Appl Phys Lett, 2004, 85: 2658-2660

17 Garnier N, Grigoriev R O, Schatz M F. Optical manipulation of microscale fluid flow. Phys Rev Lett, 2003, 91: 054501

18 Aussillous P, Quéré D. Liquid marbles. Nature, 2001, 411: 924-927

19 Fialkowski M, Bitner A, Grzybowski B A. Self-assembly of polymeric microspheres of complex internal structures. Nat Mater, 2005, 4: 93-97

20 Quéré D, Ajdari A. Liquid drops surfing the hot spot. Nat Mater, 2006, 5: 429-430

21 Madou M. Fundamentals of Microfabrication. Boca Raton: CRC, 1997

22 Bernarain J D, Stebbins C J, Mudawar I. Effects of surface roughness on water droplet impact history and heat transfer regimes. Int J Heat Mass Transfer, 1997, 40: 73-88

Open Access This article is distributed under the terms of the Creative Commons Attribution License which permits any use, distribution, and reproduction in any medium, provided the original author(s) and source are credited. 